\title{
Bacteriemia en daño hepático crónico
}

\author{
José M. Munita S., Rafael Araos B., Jorge Pérez G., Alejandra Álvarez V. , Magdalena Canals C., \\ Jorge Contreras B., Alejandra Marcotti S., Luis Thompson M. y Luis M. Noriega R.
}

\section{Bacteremia in patients with liver cirrhosis}

Bacteremic infections are more frequent in patients with cirrhosis, as their immune system is compromised. Series of cirrhotic patients with bacteremia has seldom been reported in Chile. We retrospectively collected, from 2005 to 2008, 59 episodes of bacteremia in cirrhotics representing $9 \%$ of the overall number of bacteremic episodes seen in our center in the period. Spontaneous bacteremia accounted for $29 \%$ followed by those of pulmonary origin (22\%). Grampositive cocci and gramnegative bacilli were responsible in $52 \%$ and $48 \%$ respectively, however gramnegative rods predominated in nosocomial bacteremias. Overall, the most frequent organisms were Staphylococcus aureus (24\%) and Escherichia coli (22\%). Mortality in bacteremic patients was significantly higher compared with all cirrhotic patients hospitalized in the period (37.0 vs 9.4\%; p < 0.001) and MELD score was significantly correlated with mortality. Conclusion: bacteremia is a severe complication of cirrhosis and MELD score could be a useful tool to stratify risk in these patients.

Key words: Liver cirrhosis, chronic liver failure, bacteremia.

Palabras clave: Cirrosis, daño hepático crónico, bacteriemia.

\section{Introducción}

as infecciones son una de las principales causas de descompensación, y en general de morbimortalidad, en pacientes con daño hepático crónico $(\mathrm{DHC})^{1}$. Los cuadros más habituales son: peritonitis bacteriana espontánea (PBE), infección del tracto urinario (ITU), neumonía y bacteriemia $\sin$ foco ${ }^{2}$.

Se ha estimado que el riesgo de bacteriemia en un paciente con $\mathrm{DHC}$ es 10 veces mayor que el de la población general. Algunas series han reportado que la incidencia de bacteriemia en este grupo de pacientes llega a $12 \%$, siendo ésta muchas veces de origen desconocido. La incidencia es mayor en el grupo de pacientes con daño hepático avanzado y con enfermedad descompensada ${ }^{3,4}$.

Los principales agentes etiológicos de las bacteriemias varían según las distintas series, siendo los bacilos gramnegativos los agentes más relevantes ${ }^{2,5}$. No obstante, estudios recientes han reportado un aumento en la frecuencia de aislamiento de cocáceas grampositivas ${ }^{4}$, hecho que podría estar relacionado con una mayor tendencia a realizar procedimientos invasores en los pacientes que se hospitalizan ${ }^{6}$ y con el uso de profilaxis antimicrobiana contra agentes de la familia Enterobacteriaceae ${ }^{7}$. Además se ha descrito asociación entre cirrosis y bacteriemia por microorganismos específicos, como es el caso de Streptococcus pneumoniae y Streptococcus agalactiae, entre otros ${ }^{8}$.

Desde un punto de vista fisiopatológico hay diversos factores que explican, al menos en parte, la mayor susceptibilidad que presentan estos pacientes a las infecciones. Entre los más relevantes se encuentran: disminución de la acción bactericida del suero, menor capacidad de opsonización, bajos niveles de complemento y algunas alteraciones funcionales de los neutrófilos ${ }^{9}$. Otros factores que posiblemente determinan un mayor riesgo de infección son el consumo de alcohol y la alta frecuencia de procedimientos invasores a los que son sometidos los pacientes con DHC cuando se hospitalizan.

No existen en nuestro país series publicadas que describan pacientes cirróticos con infecciones bacteriémicas. A continuación se reporta la experiencia de un hospital terciario, universitario, con 380 camas, ubicado en la zona sur-oriente de la Región Metropolitana, Chile. El objetivo fue describir las características demográficas, clínicas y microbiológicas de pacientes internados con DHC y bacteriemia.

\section{Pacientes y Métodos}

Revisión de los registros clínicos y microbiológicos de todos los pacientes adultos ingresados en el Hospital Padre Hurtado de Santiago con diagnóstico de daño hepático crónico y un primer episodio de bacteriemia, entre enero de 2005 y diciembre de 2008. A partir del registro de hemocultivos positivos del laboratorio de microbiología, se identificaron en el sistema electrónico de epicrisis los

\author{
Hospital Padre Hurtado, \\ Santiago, Chile. \\ Departamento Medicina, (JMMS \\ $R A B, J P G, A A V, J C B)$. \\ Universidad del Desarrollo, \\ Santiago, Chile. \\ Facultad de Medicina Clínica \\ Alemana-(JMMS, RAB, JPG, AAV, \\ MCC, JCB, AMS, LTM, LMNR). \\ Clínica Alemana \\ de Santiago, Chile. \\ Departamento Medicina, (RAB, JPG, \\ AMS, LTM, LMNR). \\ Departamento de Endoscopia y \\ Enfermedades Digestivas (JCB). \\ Los autores manifiestan no \\ tener conflictos de interés. \\ Recibido: 15 de marzo de 2010 \\ Aceptado: 9 de noviembre de 2010 \\ Correspondencia a: \\ José Manuel Munita Sepúlveda \\ jmunita@alemana.cl
}


pacientes egresados con las palabras clave "cirrosis", "daño hepático crónico" y "DHC". Se obtuvieron datos demográficos, clínicos y microbiológicos del grupo de interés, así como la mortalidad intrahospitalaria del episodio de bacteriemia. Se definió cirrosis como la presencia de un examen físico con estigmas de $\mathrm{DHC}$, hallazgos típicos de laboratorio y al menos una imagen sugerente, siendo necesario cumplir dos de los criterios antes mencionados.

\begin{tabular}{|c|c|}
\hline \multicolumn{2}{|c|}{$\begin{array}{c}\text { Tabla 1. Características generales de } 59 \\
\text { pacientes con bacteriemia asociada a daño } \\
\text { hepático crónico }\end{array}$} \\
\hline Sexo M/F & $35 / 24$ \\
\hline Edad (años)* & $59(10,7)$ \\
\hline Días de hospitalización* & $22(17)$ \\
\hline Co-morbilidad & n (\%) \\
\hline - Diabetes mellitus & $25(42)$ \\
\hline - Neoplasia & $4(7)$ \\
\hline - EPOC & $4(7)$ \\
\hline Etiología del $\mathrm{DHC} C^{\ddagger}$ & n (\%) \\
\hline - Etilismo & $32(54)$ \\
\hline$-\mathrm{VHB}$ & $1(2)$ \\
\hline$-\mathrm{VHC}$ & $4(7)$ \\
\hline - EHNA & $1(2)$ \\
\hline - CBP & $1(2)$ \\
\hline - Idiopático & $18(30)$ \\
\hline CHILD A/B/C ${ }^{\ddagger}$ & $4 / 18 / 34$ \\
\hline MELD* & $19(8,4)$ \\
\hline Mortalidad (\%) & $37(22)$ \\
\hline \multicolumn{2}{|c|}{$\begin{array}{l}\text { *Valores expresados como promedio y desviación estándar. } \\
\text { EPOC: enfermedad pulmonar obstructiva crónica; VHB: virus hepa- } \\
\text { titis B, VHC: virus hepatitis C; EHNA: esteatohepatitis no alcohólica; } \\
\text { CBP: cirrosis biliar primaria. „La suma no alcanza el total de } 59 \\
\text { casos pues el dato de etiología no se encontró en dos casos y el } \\
\text { puntaje de CHILD no se logró calcular en tres de ellos. }\end{array}$} \\
\hline
\end{tabular}

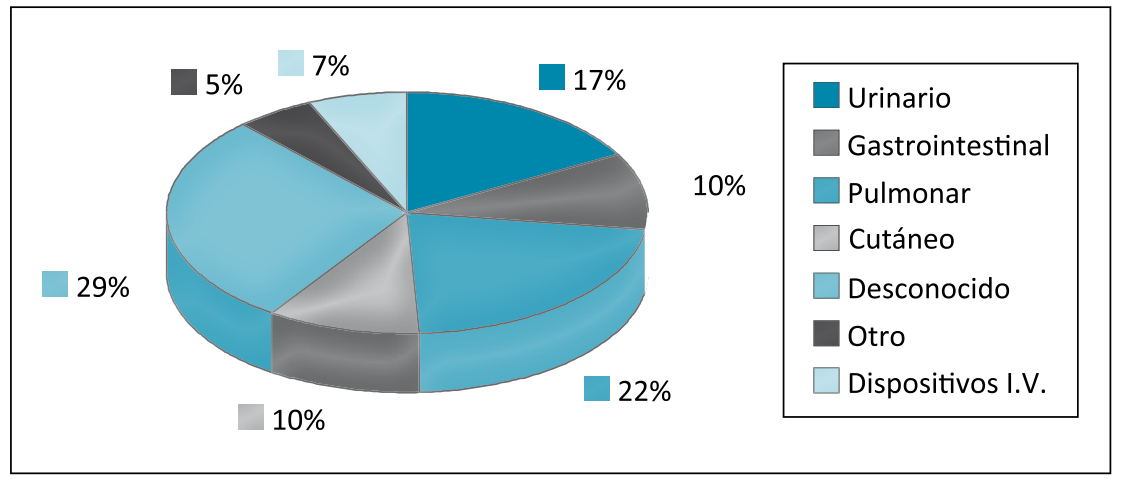

Figura 1. Frecuencia global de focos clínicos.
La causa del DHC se determinó según lo reportado por los médicos tratantes en base a los antecedentes clínicos y/o de laboratorio, y la estratificación de gravedad se basó en la escala de CHILD-PUGH ${ }^{10}$ y en el nivel de MELD (Model for End-stage Liver Diseases) ${ }^{11}$ al ingreso. Por otra parte, se definió bacteriemia como la presencia de microorganismos en al menos un hemocultivo. En el caso de Staphylococcus coagulasa negativa y Corynebacterium spp. se exigió al menos dos hemocultivos positivos. Se consideró como nosocomial aquella bacteriemia pesquisada más de 72 hrs luego de efectuado el ingreso. Todos los microorganismos aislados en sangre contaban con estudio de susceptibilidad a antimicrobianos mediante la técnica de difusión en agar ${ }^{12}$.

Las variables continuas se expresaron como promedio y desviación estándar (DS), luego de comprobar su distribución normal, y las categóricas con frecuencia y porcentaje. La comparación entre grupos fue realizada con $\mathrm{t}$ test o $\chi^{2}$ según correspondiese y se consideró significativo un $\mathrm{p}<0,05$. Para el análisis de los datos se utilizó el programa STATA 10.0 .

\section{Resultados}

Durante el período de tiempo estudiado, hubo 627 pacientes con hemocultivos positivos, de los cuales 59 $(9,4 \%)$ correspondieron a bacteriemias en pacientes con DHC. Estos correspondían al 10,4\% de 569 pacientes con DHC, que registraban un total de 831 hospitalizaciones. De las 59 bacteriemias encontradas, $35(59 \%)$ fueron en hombres. La mediana de la edad fue de 60 años (rango 33-88), sin diferencias entre sexos. Las características generales de la población se describen en la Tabla 1. La causa más frecuente de DHC fue el consumo de alcohol (32 pacientes, 54\%). Treinta y tres pacientes presentaron al menos una co-morbilidad, siendo diabetes mellitus la más común (25 pacientes, $42 \%$ ). En relación a la gravedad del DHC, el promedio del MELD fue $18(\mathrm{DS} \pm 8,4)$ y la distribución según CHILD-PUGH A/B/C fue 4/18/34.

De las bacteriemias, 31 fueron causadas por microorganismos grampositivos y 28 por gramnegativos. El foco más frecuente fue pulmonar con 13 casos $(22 \%)$, seguido del urinario con $10(17 \%)$. La bacteriemia de origen cutáneo fue más frecuente en los cuadros adquiridos en la comunidad y la secundaria a dispositivos intravasculares en los cuadros de origen nosocomial. Cabe destacar que en $17(29 \%)$ episodios no se logró identificar un foco clínico (Figura 1). El agente más frecuentemente aislado fue Staphylococcus aureus (24\%), seguido de Escherichia coli $(22 \%)$. Al analizar las bacteriemias según su procedencia, 27 (46\%) episodios fueron de origen nosocomial y $32(54 \%)$ comunitario. De estos últimos, 21 (65\%) fueron causados por agentes grampositivos, destacando 
Staphylococcus aureus y $S$. pneumoniae en 7 y 5 casos respectivamente (Tabla 2). Los microorganismos gramnegativos predominaron en las bacteriemias nosocomiales, siendo responsables de 17/27 (63\%) episodios (Tabla 3).

$\mathrm{Al}$ analizar los patrones de susceptibilidad a antimicrobianos se observó que la mayoría de las cocáceas grampositivas adquiridas en la comunidad permanecen susceptibles a antimicrobianos de uso habitual, como es el caso de $S$. aureus y $S$. pneumoniae, que fueron susceptibles a oxacilina y penicilina, respectivamente,

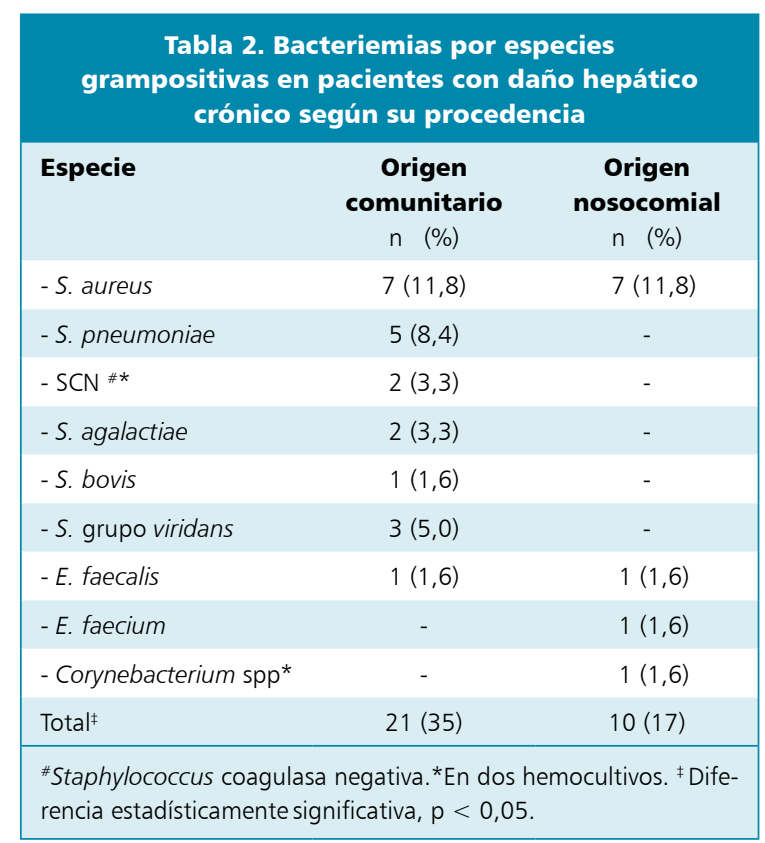

\begin{tabular}{|c|c|c|}
\hline Especie & $\begin{array}{c}\text { Origen } \\
\text { comunitario } \\
n \quad(\%)\end{array}$ & $\begin{array}{c}\text { Origen } \\
\text { nosocomial } \\
n \quad(\%)\end{array}$ \\
\hline - E. coli & $10(17)$ & $3(11,8)$ \\
\hline - C. freundii & $1(1,6)$ & - \\
\hline - K. pneumoniae & - & $4(6,7)$ \\
\hline - P. vulgaris & - & $1(1,6)$ \\
\hline - E. cloacae & - & $2(3,3)$ \\
\hline - P. aeruginosa & - & $3(5,0)$ \\
\hline - A. baumannii & - & $4(6,7)$ \\
\hline Total $^{*}$ & $11(18,6)$ & $17(28,8)$ \\
\hline
\end{tabular}

en todos los aislados. En bacteriemias de origen nosocomial, 5 de 7 cepas de $S$. aureus identificadas fueron resistentes a meticilina y de éstas, 3 de 5 fueron susceptibles a cotrimoxazol. Todas las cepas de $S$. aureus de origen nosocomial resultaron sensibles a vancomicina y rifampicina. Respecto a los bacilos gramnegativos, de 10 aislados de $E$. coli extra-hospitalaria, se encontró una cepa productora de $\beta$-lactamasa de espectro expandido (BLEE) y tres resistentes a quinolonas, siendo todas susceptibles a amikacina y carbapenémicos. La totalidad de las enterobacterias de origen nosocomial fueron productoras de BLEE y susceptibles a los carbapenémicos estudiados (ertapenem e imipenem). Se identificaron cuatro episodios de bacteriemia por Acinetobacter baumannii, siendo todos sensibles a cefoperazona/sulbactam y carbapenémicos. Por último, dos de tres aislados de Pseudomonas aeruginosa fueron susceptibles a amikacina y ciprofloxacina y las tres cepas aisladas resultaron resistentes a ceftazidima y susceptibles a carbapenémicos.

La mortalidad en el grupo de pacientes bacteriémicos con DHC fue de $37 \%$ (22/59). En la Tabla 4 se detallan las distintas variables clínicas relacionadas a la mortalidad. Luego de un análisis multivariado, encontramos una asociación estadísticamente significativa con mortalidad para las bacteriemias nosocomiales $(59 v s 18 \%, \mathrm{p}<0,002)$ y para el nivel de bilirrubinemia el día de la bacteriemia y 48 horas más tarde. La mortalidad en los pacientes con bacteriemia por microorganismos gramnegativos fue de $46 \%$ y la de aquellos con un grampositivo fue de $29 \%$, tendencia que no alcanzó significación estadística $(\mathrm{p}=$ 0,13). El MELD promedio al ingreso fue de 19 en los pacientes vivos y de 21 en el grupo de fallecidos ( $p=$ NS). El promedio de MELD a las 72 horas de hospita-

\begin{tabular}{|c|c|c|c|c|c|}
\hline & \multicolumn{2}{|c|}{ Vivos } & \multicolumn{2}{|c|}{ Fallecidos } & \multirow[b]{2}{*}{$P$} \\
\hline & Media & DS & Media & DS & \\
\hline Edad (años) & 59 & 10,7 & 60 & 11,7 & 0,7 \\
\hline INR & 2,0 & 0,87 & 2,8 & 2,6 & 0,11 \\
\hline Bilirrubinemia mg\% & 2,78 & 1,89 & 4,74 & 5,2 & 0,04 \\
\hline Albúminemia mg\% & 2,47 & 0,45 & 2,2 & 0,7 & 0,24 \\
\hline Plaquetas $/ \mathrm{mm}^{3}$ & 95.861 & 54.483 & 115.571 & 50.733 & 0,18 \\
\hline Creatininemia mg\% & 1,56 & 1,3 & 1,56 & 1,4 & 0,98 \\
\hline Natremia mEq/L & 134 & 5,9 & 136 & 6,5 & 0,15 \\
\hline MELD inicio & 19 & 8 & 20 & 9,1 & 0,5 \\
\hline MELD $72 \mathrm{~h}$ & 14,9 & 12,5 & 25,4 & 17,7 & 0,009 \\
\hline Delta MELD* & $-0,44$ & 5 & 6,15 & 11,2 & 0,007 \\
\hline
\end{tabular}


lización fue de 15 en los pacientes que sobrevivieron y de 25 en aquellos que fallecieron, diferencia que resultó estadísticamente significativa $(p<0,009)$, demostrando una buena predicción de mortalidad.

\section{Discusión}

El grupo de pacientes cirróticos reportado dio cuenta de cerca de $10 \%$ del total de las bacteriemias observadas durante el período de estudio, lo que refleja la importancia de las infecciones graves en estos pacientes. Como ha sido descrito, lo anterior podría estar favorecido por la inmunosupresión determinada por la insuficiencia hepática ${ }^{9}$ y por factores asociados a ésta como el alcoholismo ${ }^{13}$.

La serie que reportamos está compuesta fundamentalmente por pacientes con DHC avanzado, cuya principal etiología es el daño hepático por alcohol, hallazgos concordantes con la realidad chilena ${ }^{14}$.

En un alto porcentaje de los casos de bacteriemia reportados no se encontró un foco clínico, hallazgo que podría deberse a la alta frecuencia de traslocación bacteriana intestinal descrita en los pacientes cirróticos ${ }^{15,16}$. Sin embargo, cabe destacar que existen series cuyo porcentaje de ausencia de foco es menor a $10 \%$, diferencia que podría explicarse por la naturaleza retrospectiva de nuestro trabajo, lo que no permite asegurar una búsqueda sistemática del foco clínico ${ }^{17}$. En relación a los hallazgos bacteriológicos, en el análisis global no encontramos diferencias entre la frecuencia de agentes grampositivos y gramnegativos. No obstante, al comparar el tipo de microorganismo entre el grupo de pacientes con episodios de origen comunitario y nosocomiales sí hubo diferencias significativas. En el primer grupo predominaron las cocáceas grampositivas, a expensas fundamentalmente de $S$. pneumoniae y $S$. aureus, como agentes etiológicos de neumonía de la comunidad e infecciones de piel y tejidos blandos respectivamente. Lo anterior refuerza el hecho que este grupo de pacientes debiera ser vacunado contra $S$. pneumoniae ${ }^{18}$. En el grupo de bacteriemias nosocomiales fueron más frecuentes los bacilos gramnegativos multi-resistentes, hallazgo que es un reflejo de la epidemiología microbiológica de nuestro centro (datos no publicados) y que resulta concordante con los focos clínicos identificados. Por otra parte, la profilaxis antimicrobiana con quinolonas no es una práctica generalizada en nuestro centro, lo que podría explicar, al menos en parte, porqué no observamos un viraje más pronunciado hacia infecciones por especies grampositivas, hallazgo descrito en otros países ${ }^{19}$.
La mortalidad del grupo estudiado fue de $37 \%$, lo que concuerda con cifras reportadas en distintas series de la literatura médica ${ }^{17,20}$, confirmando que la presencia de bacteriemia es un factor de mal pronóstico en el paciente cirrótico hospitalizado. El diseño de este estudio no contempló la revisión del total de pacientes cirróticos infectados, por lo que no fue posible establecer la importancia relativa de la bacteriemia como factor de riesgo para mortalidad en infecciones, lo que será motivo de una nueva investigación. Observamos una mortalidad significativamente mayor para los episodios de bacteriemia de origen nosocomial. El tipo de diseño del estudio no permite determinar la validez de este dato, el que podría estar en el contexto de pacientes más graves. Hubo una tendencia hacia una mayor mortalidad en las bacteriemias por agentes gramnegativos; sin embargo, no alcanzó significación estadística.

Por último, la medición del MELD al ingreso no se correlacionó con el riesgo de morir; no obstante, su medición a las 72 horas y el delta entre ambos valores demostraron una importante asociación con mortalidad, aun luego de corregir por variables confundentes. El MELD es una medición sencilla que podría ser de gran utilidad como factor pronóstico en el paciente cirrótico con bacteriemia. La confirmación de estos hallazgos requiere la realización de un estudio prospectivo.

\section{Resumen}

Las infecciones bacteriémicas son más frecuentes en el paciente cirrótico que en la población general. Se identificaron retrospectivamente 59 pacientes cirróticos con bacteriemia, hospitalizados entre los años 2005 y 2008. La bacteriemia sin foco fue la más frecuente (29\%), seguida de aquellas de origen pulmonar (22\%). Cincuenta y dos por ciento de los agentes aislados correspondieron a cocáceas grampositivas y $48 \%$ a bacilos gramnegativos, siendo estos últimos los agentes predominantes en las bacteriemias nosocomiales. Los principales agentes aislados fueron Staphylococcus aureus $(24 \%)$ y Escherichia coli $(22 \%)$. La mortalidad de los pacientes cirróticos bacteriémicos fue mayor que la del total de cirróticos hospitalizados (37 vs 9,4\%; p < 0,001 ) y la medición del puntaje de MELD a las 72 horas se correlacionó significativamente con la mortalidad. Conclusión: La bacteriemia es una complicación grave del paciente cirrótico y el MELD podría ser útil en su categorización de riesgo. 


\section{Referencias}

1.- Tandon P, García-Tsao G. Bacterial infections, sepsis, and multiorgan failure in cirrhosis. Semin Liver Dis 2008; 28: 26-42.

2.- Fernández J, Navasa M, Gómez J, Colmenero J, Vila J, Arroyo V, et al. Bacterial infections in cirrhosis: Epidemiological changes with invasive procedures and norfloxacin prophylaxis. Hepatology 2002; 35: 140-8.

3.- Kuo C H, Changchien C S, Yang C Y, Sheen I S, Liaw Y F. Bacteremia in patients with cirrhosis of the liver. Liver 1991; 11 (6): 334-9.

4.- Thulstrup A M, Sfrensen H T, Schfnheyder H C, Møller J K, Tage-Jensen U. Population-based study of the risk and short-term prognosis for bacteremia in patients with liver cirrhosis. Clin Infect Dis 2000; 31 (6): 1357-61.

5.- Almdal T, Skinhoj P, Friis H. Bacteremia in patients suffering from cirrhosis. Infection 1986; 14: 68-70.

6.- Campillo B, Richardet J P, Kheo T, Dupeyron C. Nosocomial spontaneous bacterial peritonitis and bacteremia in cirrhotic patients: Impact of isolate type on prognosis and characteristics of infection. Clin Infect Dis 2002; 35 (1): 1-10.

7.- Campillo B, Dupeyron C, Richardet J P, Mangeney N, Leluan G. Epidemiology of severe hospital-acquired infections in patients with liver cirrhosis: Effect of long-term administration of norfloxacin. Clin Infect Dis 1998; 26 (5): 1066-70.

8.- Christou L, Pappas G, Falagas M E. Bacterial infection-related morbidity and mortality in cirrhosis. Am J Gastroenterol 2007; 102 (7): 1510-7.

9.- Fica A. Diagnóstico, Manejo y prevención de infecciones de pacientes con cirrosis hepática. Rev Chil Infect 2005; 22 (1): 63-74.

10.- Pugh R N, Murray-Lyon I M, Dawson J L, Pietroni M C, Williams R. Transection of the oesophagus for bleeding oesophageal varices. Br J Surg 1973; 60: 646.

11.- Kamath P S, Wiesner R H, Malinchoc M, Kremers W, Therneau T M, Kosberg C L, et. al. A model to predict survival in patients with end-stage liver disease. Hepatology 2001; 33 (2): 464-70.

12.- NCCLS. 2000. Performance standards for antimicrobial disk susceptibility tests; approved standard-Seventh edition. Rep. M2-A7, Wayne, Pennsylvania.

13.- Noriega LM, González P, Canals C, Michaud P. Septicemia por Streptococcus pneumoniae. Análisis de 40 casos. Rev Méd Chile 1994; 122 : 1385-92.

14.- Medina E, Kaempffer Y. Epidemiología de la cirrosis hepática en Chile. Rev Méd Chile 1974; 102: 466.
15.- Guarner C, Soriano G. Bacterial translocation and its consequences in patients with cirrhosis. Eur J Gastroenterol Hepatol 2005; 17 (1): 27-31.

16.- Aloy Duch A, Espejo Arenas E, Mauri Pont M, García Restoy E, Simó Sanahuja M, Bella Cueto F. Bacteremia in the patient with liver cirrhosis. Prospective study of 61 episodes. Enferm Infecc Microbiol Clin 1990; 8 (9):540-3.

17.- Mathurin S, Chapelet A, Spanevello V, Sayago G, Balparda C, Virga E, et al. Infections in hospitalized patients with cirrhosis. Medicina (B Aires) 2009; 69 (2): 229-38.

18.- CDC. Prevention of Pneumococcal Disease: Recommendations of the Advisory Committee on Immunization Practices (ACIP). Morbid Mortal Wkly Rep MMWR 1997; 46 (No. RR8): 1-24.

19.- Campillo B, Dupeyron C, Richardet J P. Epidemiology of hospital-acquired infections in cirrhotic patients: effect of carriage of methicillin-resistant Staphylococcus aureus and influence of previous antibiotic therapy and norfloxacin prophylaxis. Epidemiol Infect 2001; 127 (3): 443-50.

20.- Borzio M, Salerno F, Piantoni L, Cazzaniga M, Angeli P, Bissoli F, et. al. Bacterial infection in patients with advanced cirrhosis: a multicentre prospective study. Dig Liver Dis 2001; 33 (1): 41-8. 